\title{
Coordination of the Bladder Detrusor and the External Urethral Sphincter in a Rat Model of Spinal Cord Injury: Effect of Injury Severity
}

\author{
Victor Pikov ${ }^{1}$ and Jean R. Wrathall ${ }^{2}$ \\ Departments of ${ }^{1}$ Cell Biology and ${ }^{2}$ Neuroscience, Georgetown University, Washington, DC 20007
}

Recovery of urinary tract function after spinal cord injury (SCl) is important in its own right and may also serve as a model for studying mechanisms of functional recovery after injury in the CNS. Normal micturition requires coordinated activation of smooth muscle of the bladder (detrusor) and striated muscle of the external urethral sphincter (EUS) that is controlled by spinal and supraspinal circuitry. We used a clinically relevant rat model of thoracic spinal cord contusion injury to examine the effect of varying the degree of residual supraspinal connections on chronic detrusor-EUS coordination. Urodynamic evaluation at 8 weeks after $\mathrm{SCl}$ showed that detrusor contractions of the bladder recovered similarly in groups of rats injured with a 10 gm weight dropped $12.5,25$, or $50 \mathrm{~mm}$ onto the spinal cord. In contrast, the degree of coordinated activation of the EUS varied with the severity of initial injury and the degree of preservation

After incomplete spinal cord injury (SCI), both reflex and voluntary motor functions below the level of the injury are initially lost; partial recovery occurs with time after injury (Gale et al., 1985; Basso et al., 1995; Ko et al., 1999). Although the return of segmental reflexes is relatively fast, the recovery of functions mediated by supraspinally controlled reflexes is slow and limited in extent (Leis et al., 1996; Hiersemenzel et al., 2000). Because approximately one-half of the patients with spinal trauma have incomplete injury (Bracken et al., 1990), treatments to improve recovery of function after SCI are important. A primary requirement is an understanding of the mechanisms involved in the natural recovery of function after incomplete SCI.

Incomplete SCI results in an initial loss and later partial recovery of lower urinary tract (LUT) function (Pikov et al., 1998). This provides an attractive model to study mechanisms of functional recovery for several reasons. The pathways involved are relatively simple as compared, for instance, with those involved in the control of locomotion (Dietz et al., 1999). Control of LUT function has been well studied both in normal animals and animals after spinal transection (Tiseo and Yaksh, 1990; de Groat, 1995; Morrison, 1997; de Groat et al., 1998). Impairment of LUT function after SCI is a clinically important problem (Selzman and

\footnotetext{
Received July 3, 2000; revised Oct. 25, 2000; accepted Nov. 2, 2000.

This study was supported by National Institutes of Health Grants RO1NS35647-03 and RO1NS37733-01.We are grateful to Dr. Luc Jasmin for his help with viral tracing, to Dr. Vale for a kind gift of corticotropin-releasing factor antibody, to Dr. Enquist for a generous gift of pseudorabies virus antiserum, and to Dr. Stacie D. Grossman for her skillful assistance with in situ autoradiography.

Correspondence should be addressed to Dr. Jean R. Wrathall, Department of Neuroscience, Georgetown University, 3970 Reservoir Road, Northwest, Washington, DC 20007. E-mail: wrathalj@georgetown.edu.

Copyright (C) 2001 Society for Neuroscience 0270-6474/01/210559-11\$15.00/0
}

of white matter at the injury site. The $12.5 \mathrm{~mm} \mathrm{SCl}$ resulted in the sparing of $20 \%$ of the white matter at the injury site and complete recovery of detrusor-EUS coordination. In more severely injured rats, the chronic recovery of detrusor-EUS coordination was very incomplete and correlated to decreased innervation of lower motoneurons by descending control pathways and their increased levels of mRNA for glutamate receptor subunits NR2A and GluR2. These results show that the extent of recovery of detrusor-EUS coordination depends on injury severity and the degree of residual connections with brainstem control centers.

Key words: bladder; urethral sphincter; dyssynergia; electromyography; urodynamics; spinal cord contusion; serotonin; corticotropin-releasing factor; glutamate receptors
Hampel, 1993). Clinical data indicate that the recovery of voluntary control of external urethral sphincter (EUS) function after incomplete SCI occurs in the same time frame as the recovery of voluntary control of somatic skeletal muscle (Schurch, 1999) and thus likely involves some of the same basic mechanisms.

Normal LUT function involves spinal and supraspinal circuitry that controls urine storage and release (de Groat, 1990). In rats, urine release is mediated by contraction of the bladder detrusor accompanied by coordinated activation of the EUS. If EUS activity is removed by neuromuscular blockade, efficient voiding is abolished (Maggi et al., 1986a; Mersdorf et al., 1993; Kakizaki et al., 1997). After thoracic spinal cord transection, there is an initial loss of bladder contractions followed by their recovery (Tiseo and Yaksh, 1990; Pikov et al., 1998). Loss of coordinated EUS activation is, however, permanent (Kruse et al., 1993; Pikov et al., 1998), indicating that pathways to and from centers above the thoracic spinal cord are important for EUS function. In a previous study of incomplete SCI (Pikov et al., 1998), we found evidence of detrusor-EUS coordination in week 2 after injury. Transneuronal viral tracing indicated that the SCI animals with recovered detrusor-EUS coordination had spared residual connections from the bladder to brainstem centers that normally are involved in micturition control including Barrington's nucleus (Kruse et al., 1990). We postulated that the presence of residual connections with brainstem control centers for micturition served as the anatomical basis of partial functional recovery. In the current study we examined this hypothesis by experimentally varying the loss of supraspinal connections by altering injury severity and thus loss of white matter at the injury site. 


\section{MATERIALS AND METHODS}

Experimental groups. Adult female Sprague Dawley rats (Zivic Miller, Allison, PA) weighing 270-350 gm were used. Rats were anesthetized with chloral hydrate $(360 \mathrm{mg} / \mathrm{kg}$, i.p.). Laminectomy was performed at T8, and SCI was produced with a weight-drop injury device (Gruner, 1992) by use of the $10 \mathrm{gm}$ weight dropped from the height of $12.5 \mathrm{~mm}$ $(n=7), 25 \mathrm{~mm}(n=5)$, or $50 \mathrm{~mm}(n=7)$ onto exposed dura. One group of control animals had laminectomies but was left uninjured $(n=6)$.

Animal care. Rats were housed in the Department of Comparative Medicine Animal Facility, where they were kept in a room with controlled humidity, temperature, and light cycle ( $12 \mathrm{hr}$ on/12 hr off) and had access to food and water ad libitum. This facility is supervised by a licensed veterinarian, meets all National Institutes of Health guidelines for the care of laboratory rodents, and is fully accredited by the Association for Accreditation of Laboratory Animal Care. Postoperatively, rats were housed in pairs (to reduce stress from isolation) and kept at $22-25^{\circ} \mathrm{C}$ on highly absorbent bedding. They received manual expression of the bladder twice daily for 2 weeks after SCI. Under these conditions, the mortality of spinal cord-injured rats was $\sim 5 \%$ ( 1 of 19 ), and no evidence of infection, pressure sores, or self-mutilation was seen in surviving animals. The one SCI animal (from the $25 \mathrm{~mm}$ group) died as a result of a urinary tract infection.

Behavioral tests of hindlimb functional deficits. All rats were tested blindly for functional deficits on days 1 and 7 and weekly thereafter through 8 weeks after injury. The combined behavioral score (CBS) was used as a measure of overall hindlimb functional deficit (Gale et al., 1985; Kerasidis et al., 1987). To calculate the CBS, we used a battery of reflex tests including toe spread and placing and withdrawal in response to stimulation, righting, and hot plate. Rats were also tested for coordination between forelimbs and hindlimbs and weight support during walking, swimming, and standing on an inclined plane. The CBS ranges from 0 to 100 , with 0 indicating no functional deficit and 100 indicating abnormal responses in all of the tests. Rats were also evaluated by an expanded open-field locomotion scale (BBB, Basso et al., 1995), in which a completely paralyzed rat scores 0 , a rat with increasing joint movements but without weight support scores between 1 and 8 , an abnormally locomoting rat with weight support scores between 9 and 20, and a normal rat scores 21 .

Assessment of LUT function. LUT function was examined using a urodynamic procedure (Maggi et al., 1986b) that allows a rapid collection of data over a large number of voiding cycles. Animals were randomly divided into blocks comprising one normal rat and rats from two or three SCI groups. Animals from each block $(n=6)$ were studied urodynamically on the same day. Bladder intravesical pressure was recorded with a transurethral bladder catheter (polyethylene-50) during continuous perfusion with warm saline $(0.22 \mathrm{ml} / \mathrm{min})$. During the bladder detrusor contractions, fluid was released by flowing around the catheter in the urethra. The signal from the pressure transducer was amplified (TA200 transducer amplifier; Caldwell Biomedical Electronics, Tully, NY), sampled at $1 \mathrm{kHz}$ using a 12-bit analog-to-digital board (model PCI-MIO16E-4; National Instruments, Austin, TX), and acquired on-line using BioBench 1.0 software (National Instruments). For electromyography $($ EMG), two fine $(50 \mu \mathrm{m})$ epoxy-coated platinum-iridium wire electrodes (A-M Systems, Inc., Everett, WA) were placed percutaneously in the sphincter area of the urethra to record EUS electrical activity. A 30 gauge needle with a hooked wire electrode positioned at the tip was inserted into the EUS and then withdrawn leaving the wires embedded in the muscle. The EMG activity was preamplified (Cornerstone AMP-8 preamplifier; Dagan Corporation, Minneapolis, MN), sampled at $1 \mathrm{kHz}$, and acquired on-line simultaneously with intravesical pressure. Although the baseline amplitude of large-skeletal muscle EMGs is a relatively consistent value, sphincteric EMG amplitude depends on the placement of the thin wire electrodes, which can cause significant variation in baseline amplitude values depending on their location (Callsen-Cencic and Mense, 1998). Therefore, the baseline fluctuations caused by a low-frequency component of the EMG (which is generally attributed to activity of the internal urethral sphincter muscle) were filtered out by the use of a baseline zero correction at $100 \mathrm{msec}$ intervals. The rate of spiking EUS activity was calculated from $60 \mathrm{~Hz}$-filtered EMG data by the use of a custom-written peak detection macro for Excel (Microsoft Corporation, Redmond, WA), which counts the number of peaks above the defined threshold at $100 \mathrm{msec}$ intervals. The average values of spiking EUS activity during bladder filling and voiding were calculated, and threshold (at the initiation of contraction) and maximal intravesical pressures during voiding were measured for each voiding cycle over a 20 min period in each of the animals.

Intravesical catheter and EMG wires were inserted while the animal was anesthetized with chloral hydrate $(360 \mathrm{mg} / \mathrm{kg}$, i.p.). The rat was then placed in a body-shaped cloth glove and allowed to recover from anesthesia for $2 \mathrm{hr}$. The urodynamic recordings were done on the awake restrained animal because anesthesia markedly reduces the efficiency of voiding (Yoshiyama et al., 1994a, 1999). After the urodynamic experiment, the animal was reanesthetized with chloral hydrate and perfused with saline followed with $4 \%$ paraformaldehyde. The bladder was removed, blot-dried, weighed, and measured. The bladder volume $(V)$ was calculated by the equation for an ellipsoid: $V=4 / 3 * \pi * L * W * W$, where $L$ is bladder length and $W$ is bladder width. This method estimates the volume of the nonexpanded bladder and therefore is possibly underestimating the real bladder volume because of elastic distension.

White matter sparing at the injury site. Spinal cord tissue was dissected and post-fixed overnight. Fifteen-millimeter-long thoracic spinal cord segments containing the injury site from two to three SCI groups and a corresponding segment from the uninjured control, comprising the block of animals from a day's urodynamic study, were embedded together in ornithine carbamyl transferase (OCT; Tissue-Tek; Sakura Finetek, Torrance, CA) and sectioned at $20 \mu \mathrm{m}$ on a cryostat (Jung Frigocut $2800 \mathrm{E}$; Leica, Deerfield, IL). One slide per millimeter length of spinal cord was stained with eriochrome cyanine for myelinated white matter (Clark, 1981). One section on the slide representing the injury epicenter (containing the least white matter) was used to quantify the area of myelinated white matter in the lateral and ventral funiculi by calculating the area covered by dark pixels (above the set threshold) with the aid of an image analysis system (Scion Image; Scion Corporation, Frederick, MD).

Analysis of motoneurons in the dorsolateral and sacral parasympathetic nuclei. L5-S2 spinal cord from the rats was used for immunohistochemical and in situ hybridization studies. Tissue segments from the uninjured control and two to three SCI groups were frozen together in OCT, and serial sections were cut at $12 \mu \mathrm{m}$ on a cryostat. One slide per millimeter length of cord was stained with neutral red, and the dorsolateral (DL) and sacral parasympathetic nuclei (SPN) were identified on the basis of criteria developed from separate animals that were used for retrograde tracing from the bladder and EUS, as described below.

Identification of DL motoneurons by retrograde labeling with Fluororuby. Multiple (six to eight) microinjections of $50 \mu \mathrm{l}$ of $10 \%$ Fluororuby (tetramethylrhodamine-labeled dextran; 3000 molecular weight; Molecular Probes, Eugene, OR) into the EUS area were given to uninjured animals $(n=2)$ using a Hamilton microsyringe. Six days after injection, the animals were anesthetized with chloral hydrate; their spinal cords were removed and fixed with $4 \%$ paraformaldehyde. Twenty micrometer cryosections from the lumbosacral spinal cord were prepared and counterstained with neutral red or the nuclear dye Hoechst 33342 (Sigma, St. Louis, MO; 1:5000) to visualize cell bodies. Sections were examined using epifluorescent illumination and appropriate filters. Drawings of the representative spinal cord profiles with labeled and nonlabeled ventral horn motoneurons were made based on results from three consecutive sections.

Identification of SPN by viral tracing. Residual brainstem-spinal projections were examined using retrograde trans-synaptic labeling with pseudorabies virus injected into the bladder wall of uninjured animals as described previously (Pikov et al., 1998). In brief, the animals were anesthetized, and pseudorabies virus (Bartha $\mathrm{K}$ strain; $\sim 15 \mu \mathrm{l}$ at $10^{9}$ $\mathrm{pfu} / \mathrm{ml}$ ) was slowly injected into the ventral bladder detrusor muscle. Three to $4 \mathrm{~d}$ later, rats were reanesthetized and perfused with fixative, and frozen sections were prepared. The location of the virus in the SPN of S1 spinal cord was confirmed by immunohistochemistry using an anti-pseudorabies virus rabbit polyclonal antiserum (serum Rb-134; a gift from Dr. Lynn Enquist, Princeton University, Princeton, NJ; 1:20,000).

Immunohistochemistry. Sections through the middle of DL were incubated with a rabbit anti-rat polyclonal antibody to serotonin (\#20080; Diasorin, Stillwater, MN; 1:100). Sections containing SPN were incubated with a rabbit antiserum to rat/human corticotropin-releasing factor (CRF; a gift from Dr. W. Vale, The Salk Institute, La Jolla, CA; 1:2000). Immunoreactivity was visualized using biotin-conjugated secondary antibodies (Vector Laboratories, Burlingame, CA) and the peroxidase chromogen SG (Vector Laboratories). Each slide contained the sections from the uninjured control and all experimental groups. One slide for SPN and one for DL were analyzed for each animal. Three nonconsecutive sections were selected from each slide, and the immunoreactivity was quantified for both the left and the right nucleus using an 


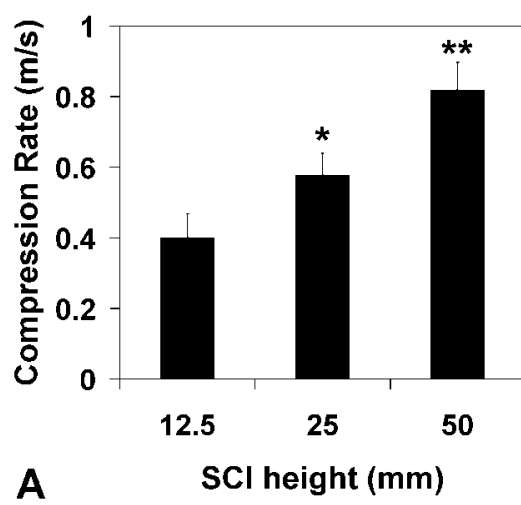

A

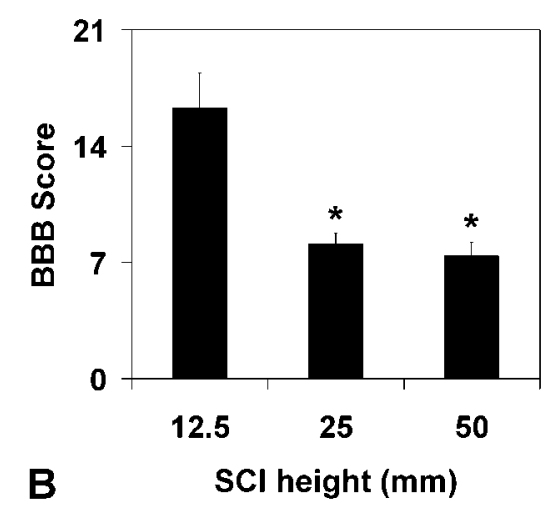

B

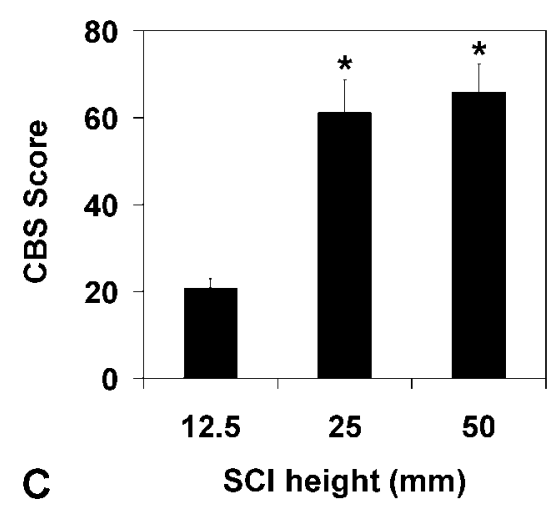

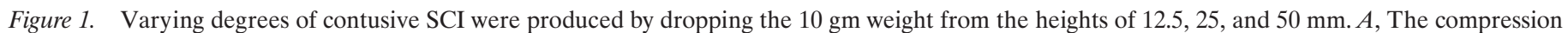

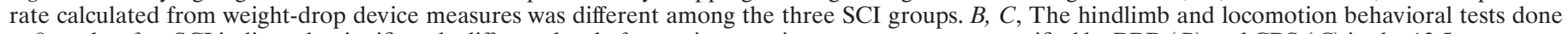

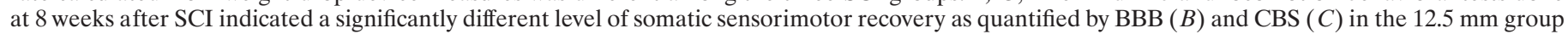

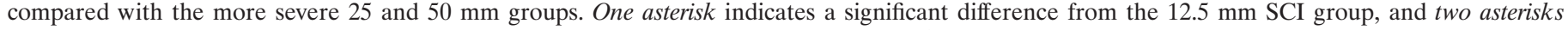
indicate a difference between 25 and $50 \mathrm{~mm}$ groups, based on $p<0.05$ in Tukey's post hoc test after ANOVA.

image analysis program (Scion Image) to calculate an average value for that animal. CRF immunoreactivity in the SPN was quantified by capturing the area of the nucleus $(260 \times 230 \mu \mathrm{m})$ and counting the number of dark pixels in that area. Quantification of the whole area rather than that around individual motoneurons was performed because of the high density and small size of the SPN neurons. Serotonin immunoreactivity surrounding DL motoneurons was quantified by tracing the cell boundary and counting the number of dark pixels (above the set threshold) outside of the cell body and within a circle (68 $\mu \mathrm{m}$ in diameter) centered on the cell body. If the sampling circles of adjacent motoneurons were overlapping, the serotonin immunoreactivity around these motoneurons was not quantified. This resulted in the exclusion of $<10 \%$ of motoneurons. The average density of serotonin immunoreactivity for DL motoneurons was based on analysis of 10-29 neurons per animal. The average animal values were used in the statistical analyses.

In situ hybridization. Slides containing sections through the DL nucleus from the uninjured controls and two to three SCI groups were hybridized with ${ }^{35}$ S-dATP-labeled antisense oligonucleotides to NMDA (NR1, NR2A, and NR2B) and AMPA (GluR1, GluR2, GluR3, and GluR4) subunit mRNA, as described previously (Grossman et al., 1999, 2000). Slides were exposed to Eastman Kodak (Rochester, NY) NT2B photographic emulsion for 7 weeks and then developed (with D19 developer), fixed with general-purpose fixer (Eastman Kodak), and counterstained with neutral red. Grain counting was performed by tracing neutral red-stained cell bodies and counting the cell size and number of overlying grains by use of an image analysis program (Scion Image). The density of grains (grains per square micrometer) for a given cell was calculated as the ratio of the grain number to the cell size and corrected for background by subtracting the density of grains in adjacent areas devoid of tissue. DL motoneurons on both sides of two nonconsecutive sections on each of two slides were examined in each animal (15-32 in total), and the mean animal values were used in statistical analysis. In addition, the number of DL motoneurons per section was determined for each animal.

Statistical analysis. All data were subjected to statistical analysis using the SigmaStat 2.0 program (SPSS, Inc., Chicago, IL). One-way ANOVA or repeat measures ANOVA tests were the main tests used, followed by post hoc tests (Tukey) of differences between specific groups or time points. A significance level of $p<0.05$ was used. Throughout the text, figures, and tables, the mean value \pm SD notations are used in describing the results, unless otherwise indicated.

\section{RESULTS}

\section{Severity of spinal cord injury}

At the time of contusion, the readings from the weight-drop apparatus provided information about the velocity of impact and the extent and duration of spinal cord compression (Gruner, 1992). From these data, the compression rate was calculated as the ratio of cord compression depth over time because this parameter was established previously to be the most sensitive measure of the initial impact (Constantini and Young, 1994). The average compression rate was found to be significantly different among the three SCI groups (Fig. 1A).

Behavioral tests of hindlimb function were done during the recovery period after SCI (day 1 and weekly through 8 weeks). As described previously (Gale et al., 1985; Basso et al., 1996), hindlimb functional deficits were maximal at day 1 and then showed partial recovery over the next several weeks with a plateau seen by 3 weeks after injury. At day 1 the three injury groups were indistinguishable, but by 1 week the $12.5 \mathrm{~mm}$ group demonstrated increased recovery compared with the 25 and $50 \mathrm{~mm}$ groups (data not shown). Hindlimb functional recovery in the small number of rats in the $25 \mathrm{~mm}$ group ( $n=4)$ was not significantly different from that in the $50 \mathrm{~mm}$ group at any week after injury, although a nonsignificant trend toward enhanced recovery was seen on weeks 3-8. Chronic (week 8) behavioral measures of overall hindlimb functional deficits (Fig. $1 C, \mathrm{CBS}$ ) and of locomotion recovery (Fig. $1 B$, BBB) showed significant differences between the $12.5 \mathrm{~mm}$ group, which attained weight-bearing locomotion, and the 25 and $50 \mathrm{~mm}$ groups, which on average did not. This is an important distinction, because voluntary overground locomotion with full weight support requires supraspinal control (Grillner and Dubuc, 1988; Mori et al., 1999).

After the behavioral and urodynamic evaluations on week 8, the chronic injury sites were examined for histopathology. As described previously for this and other models of contusion injury in the rat (Noble and Wrathall, 1985; Basso et al., 1996; Beattie et al., 1997), cross sections of the injury epicenter (region of maximal damage) demonstrated a central lesion devoid of normal spinal cord tissue surrounded by a peripheral rim of preserved white matter (Fig. 2). White matter immediately adjacent to the central lesion appeared very lightly stained. More peripherally located white matter was closer to normal in its eriochrome cyanine staining. On the basis of a previous evaluation of residual white matter 8 weeks after SCI at the electron microscopy level (Wrathall et al., 1998), we interpreted the reduced myelin staining to be caused by a combination of axonal loss, which is greatest near the central cavity, as well as hypomyelination of the surviving axons. The amount of this residual myelinated white matter appeared to be less in the more severe injury groups. We quan- 
Figure 2. White matter sparing at the SCI epicenter. Top, Bottom Left, Photomicrographs of representative sections through the lesion epicenter from the three SCI groups and from an uninjured control stained with eriochrome cyanine to label myelin. The dorsal, lateral, and ventral funicular white matter of the normal spinal cord is heavily stained, whereas little myelin staining is seen in the central gray matter. The cross-sectional profiles of the injured spinal cords are reduced in diameter. The center of the injured cords contains cavities and an abnormal loose network of cells, but no myelin staining is apparent. A peripheral rim of residual white matter is seen. Myelin staining is present but reduced compared with normal white matter, consistent with the chronic hypomyelination of residual axons. Bottom Right, The average areas of myelinated white matter from the ventral and lateral funicular zones at the lesion epicenter in the SCI groups. One asterisk indicates a significant difference from the $12.5 \mathrm{~mm} \mathrm{SCI}$ group, based on $p<0.05$ in Tukey's post hoc test after ANOVA. Scale bar, $250 \mu \mathrm{m}$.
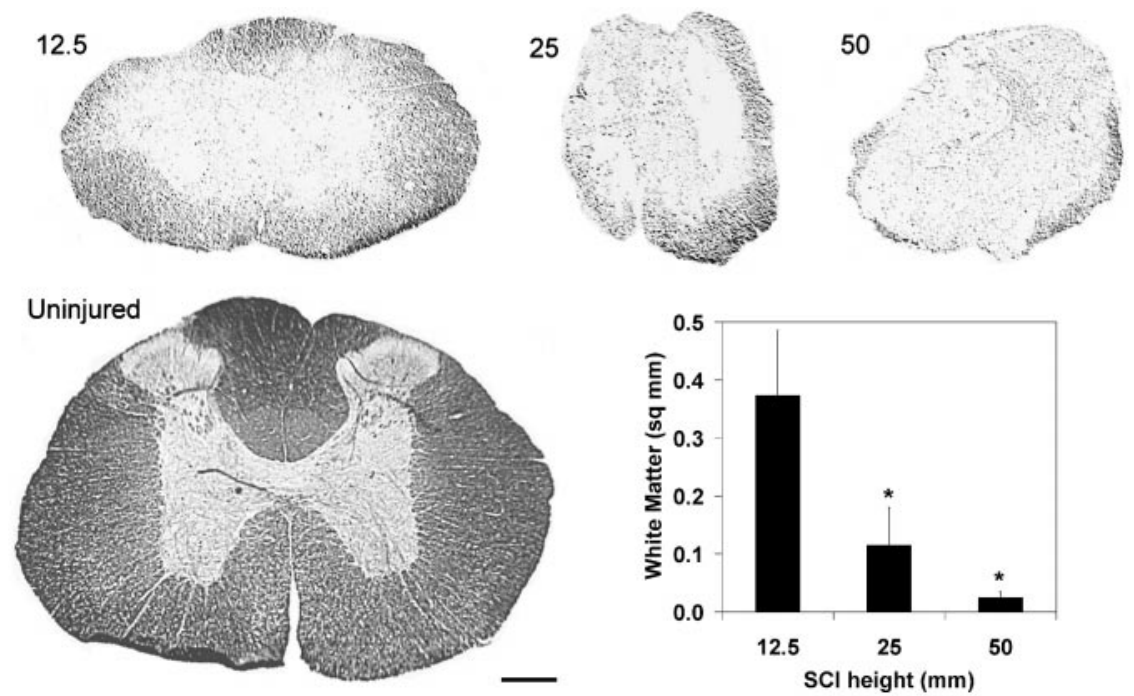

tified the area of residual white matter in lateral and ventral funiculi at the injury epicenter (Fig. 2, bottom right), because these funiculi have been shown previously to contain both the ascending and descending micturition-related pathways (Loewy et al., 1979; Fedirchuk and Shefchyk, 1991). The $12.5 \mathrm{~mm}$ group retained significantly greater residual white matter than did the more severely injured groups. They had more than triple the residual white matter of the $25 \mathrm{~mm}$ group and $\sim 10$ times that of the $50 \mathrm{~mm}$ group. The residual white matter in the lateral and ventral funiculi of the $12.5 \mathrm{~mm}$ group was $\sim 20 \%$ of that in uninjured control rats. Taken together, the biomechanical, behavioral, and morphometric measures of SCI severity indicate that the $12.5 \mathrm{~mm}$ group of animals sustained significantly less initial traumatic injury and exhibited less neurological impairment and chronic white matter loss at the lesion site than did the 25 and 50 mm groups.

\section{Effect of injury severity on spontaneous LUT function}

Initially after SCI the rats were not capable of spontaneous micturition, and their bladders were manually expressed twice daily. The volume of expressed urine was measured each time, and the data were used to estimate the initiation of spontaneous LUT function after SCI. In all rats the volume of manually expressed urine increased over the first few days after SCI and then decreased as spontaneous micturition was reestablished. On the basis of our previous data on increased bladder size with time after SCI (Pikov et al., 1998) and a limited study using metabolic cages (V. Pikov, unpublished observations), the increase in volume of expressed urine during the first $4 \mathrm{~d}$ after injury was interpreted as resulting from increased bladder size in the absence of spontaneous micturition. The subsequent decrease in manually expressed urine was interpreted to indicate the initiation of spontaneous micturition. Because of the maintenance of an enlarged bladder and the reduced voiding efficiency of SCI rats (Pikov et al., 1998), the volume of urine that could be expressed remained higher than that in uninjured rats even after spontaneous micturition was established. This was especially evident in the 25 and $50 \mathrm{~mm}$ groups. Thus, the injury groups demonstrated differences in that the more severely injured rats retained a higher residual urine volume and required a longer time period before displaying evidence of spontaneous micturition (Fig. 3). The average time for initiation of spontaneous micturition was signif-

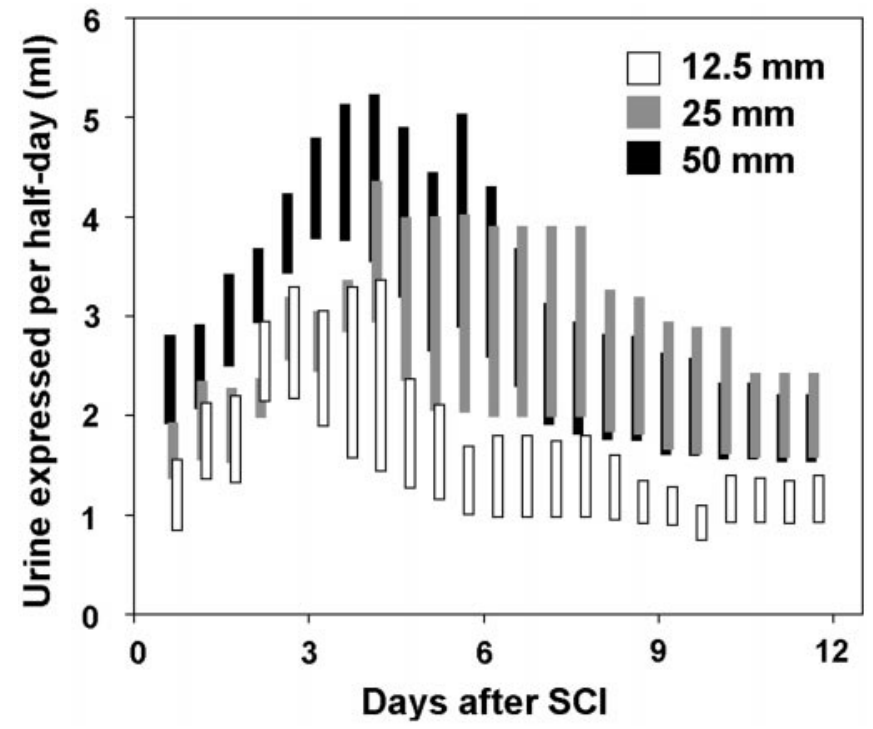

Figure 3. Time course of recovery of spontaneous voiding. Urinary bladders were expressed every $12 \mathrm{hr}$, and the collected urine volume was measured. The height of the bars indicates urine volume within one SE above and below the group mean at the specified time point. Compared with 25 and $50 \mathrm{~mm}$ SCI animals, the $12.5 \mathrm{~mm}$ SCI animals had progressively less urine collected with time after SCI.

icantly longer in the $50 \mathrm{~mm}$ group than in the $12.5 \mathrm{~mm}$ group (Table 1). Chronically, at 8 weeks after SCI, bladder weights and volumes were significantly increased in the 25 and $50 \mathrm{~mm}$ groups compared with uninjured controls (Table 1).

\section{Effect of injury severity on chronic urodynamic function}

Urodynamic evaluation chronically at 8 weeks after SCI demonstrated that injury severity was correlated with the degree of recovery of detrusor-EUS coordination. Uninjured animals had large bladder detrusor contractions (Fig. 4A; Table 1), which were accompanied by increased amplitude in EUS EMG (Fig. 4D). The bladder contraction often had two incremental pressure increases, with the first intravesical pressure increase corresponding to a maximal increase in EUS activity and extensive voiding. A second increase in intravesical pressure (overshoot) was often 
Table 1. Changes in LUT parameters after SCI

\begin{tabular}{lcccc} 
& SCI $(\mathrm{mm})$ & & \\
\cline { 2 - 5 } Parameter & $0(n=6)$ & $12.5(n=7)$ & $25(n=4)$ & $50(n=7)$ \\
\hline Spontaneous voiding (dpi) & $0.0 \pm 0.0$ & $2.5^{*} \pm 1.8$ & $4.3^{*} \pm 1.0$ & $4.4^{* *} \pm 0.9$ \\
Bladder weight $(\mathrm{gm})$ & $0.11 \pm 0.01$ & $0.23 \pm 0.09$ & $0.37^{*} \pm 0.13$ & $0.41^{*} \pm 0.18$ \\
Bladder volume $(\mathrm{ml})$ & $3.8 \pm 1.5$ & $9.1 \pm 6.0$ & $14.9^{*} \pm 6.2$ & $26.1^{* *} \pm 8.1$ \\
Amplitude (mmHg) & $25.4 \pm 7.7$ & $19.4 \pm 7.6$ & $14.1 \pm 3.0$ & $13.0^{*} \pm 5.1$
\end{tabular}

The number of animals in each group is shown in parentheses. Spontaneous voiding was calculated as the number of days postinjury (dpi) when the volume of manually expressed urine first began to decrease. Urodynamic evaluation was performed at 8 weeks after surgery in SCI $(12.5,25$, and $50 \mathrm{~mm})$ and uninjured $(0 \mathrm{~mm})$ groups. The bladder was then weighed after blot-drying, and its length and width were measured to calculate the volume. The amplitude of bladder pressure was calculated as the difference between the maximal intravesical pressure during voiding and the pressure just before the voiding was initiated (threshold pressure). There was a significant difference among the SCI groups as calculated by ANOVA ( $p=$ $0.01)$, with Tukey's post hoc test indicating the significant difference $(p<0.05)$ from the uninjured group (one asterisk) or from the uninjured and $12.5 \mathrm{~mm}$ groups (two asterisks).

seen and coincided with cessation of EUS activation and little or no voiding. Unlike uninjured animals, all SCI animals lacked the intravesical pressure overshoot and demonstrated somewhat decreased bladder detrusor contractions in response to bladder filling (Fig. 4B,C; Table 1).

The time-frequency analysis of EUS EMG yielded important quantitative parameters, such as mean EMG activity, mean EMG power frequency, and mean EMG-spiking activity (ESA) (Fig. 4). The timing of the coordinated EUS activation was unaltered by the SCI (data not shown) in the animals in which a detectable increase in EMG parameters was found. The EMG parameters were compared between the contraction and filling phases. All of the rats in the $12.5 \mathrm{~mm}$ SCI group exhibited bladder contractions leading to stream-like voiding (Fig. $4 B$ ) and an increase in the
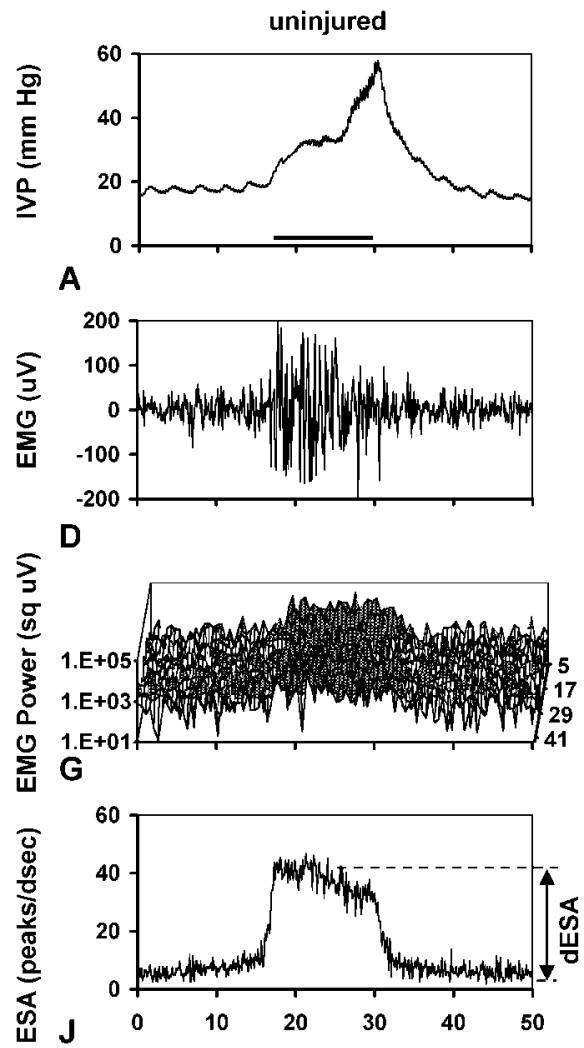

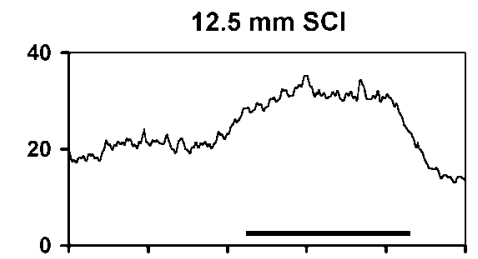

B

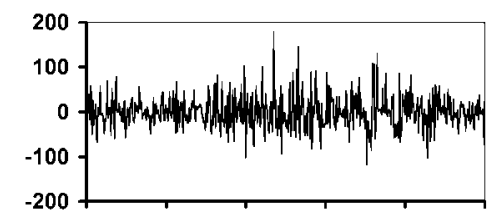

E
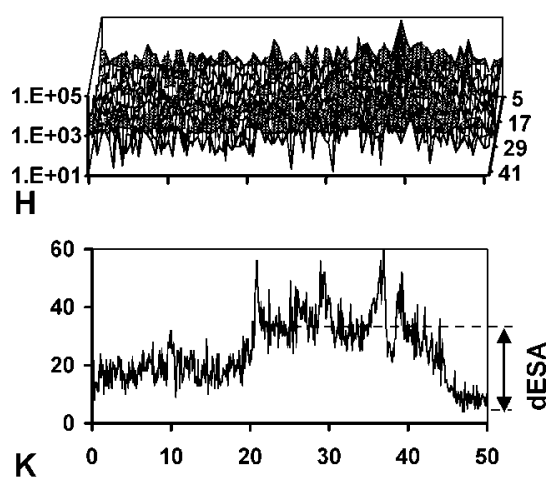

C
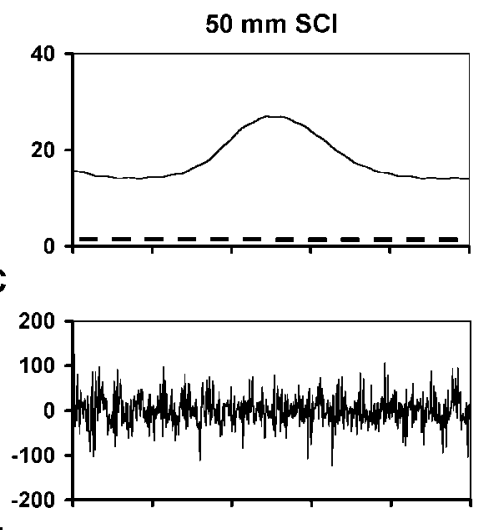

$\mathbf{F}$
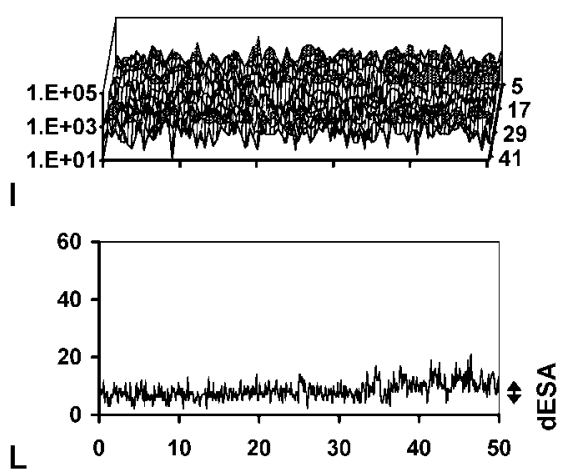

time (sec)

Figure 4. Urodynamic analysis of detrusor-EUS coordination in representative uninjured $(A, D, G, J), 12.5 \mathrm{~mm} \mathrm{SCI}(B, E, H, K)$, and $50 \mathrm{~mm} \mathrm{SCI}(C$, $F, I, L)$ animals. $A-C$, Bladder intravesical pressure $(I V P)$ recordings during one voiding cycle. Solid horizontal lines in $A$ and $B$ indicate the duration of stream-like voiding, and the dashed line in $C$ indicates the drop-by-drop voiding. $D-F$, EUS EMG recordings, showing an activation of EUS EMG in relationship to the voiding cycle in uninjured and $12.5 \mathrm{~mm} \mathrm{SCI}$ animals but not in the $50 \mathrm{~mm}$ SCI animal. $G-I$, Power spectrum analysis of EUS EMG activity as a function of time. A broad band of frequencies $(5-40 \mathrm{~Hz})$ shows an increased power during the voiding phase in uninjured and $12.5 \mathrm{~mm}$ SCI animals but not in the $50 \mathrm{~mm}$ SCI animal. $J-L$, Peak detection analysis of the ESA. Peaks were detected in 1 dsec intervals. An increase in ESA (dESA) occurred at the time corresponding to the voiding phase in uninjured and $12.5 \mathrm{~mm} \mathrm{SCI}$ animals, but there was no change in the level of ESA in the 50 mm SCI animal. 

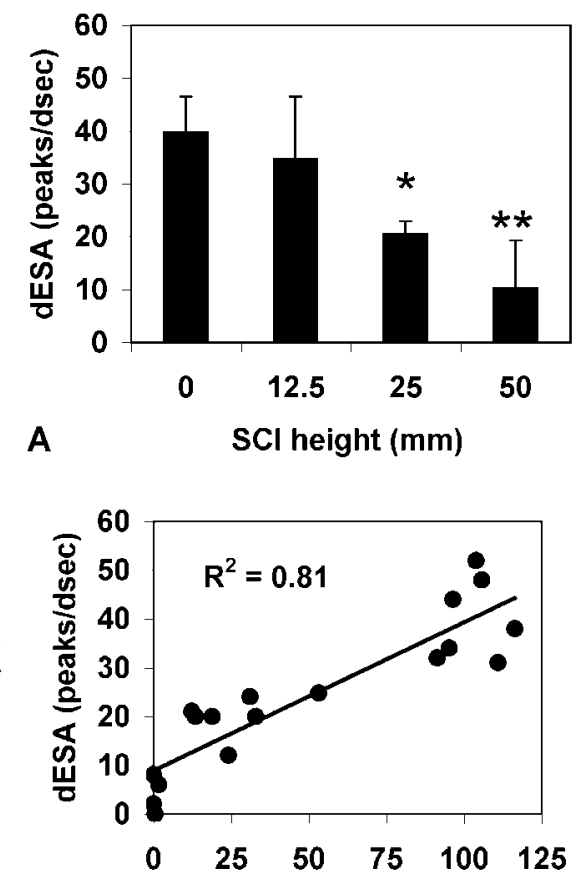

C $\quad 5 \mathrm{HT}$-ir at DL (\% uninjured)
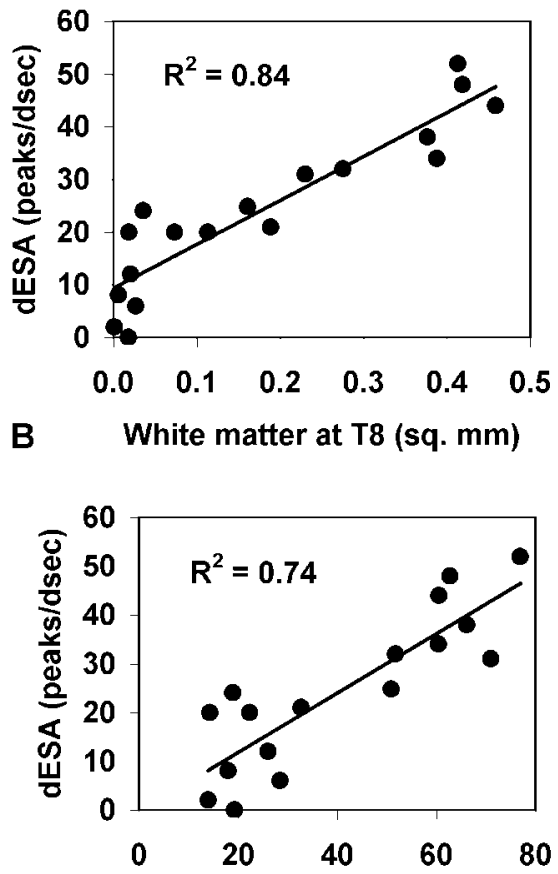

D

CRF-ir at SPN (\% uninjured) amplitude of EUS activity at the time of detrusor contractions (Fig. $4 E$ ). In contrast, in the animals from the 25 and $50 \mathrm{~mm}$ groups, the urine was released in a dropwise manner independent of detrusor contractions (Fig. 4C). Animals in these groups showed little or no increase in EUS EMG amplitude during detrusor contractions (Fig. $4 F$ ).

Changes in EUS EMG were evaluated during the progression of the micturition cycle by power spectrum analysis using the fast Fourier transform algorithm (Fig. 4G-I). During the voiding phase as compared with the filling phase, the power of the 5-40 $\mathrm{Hz}$ EMG frequency band was increased in both uninjured (Fig. $4 G$ ) and $12.5 \mathrm{~mm} \mathrm{SCI}$ animals (Fig. $4 H$ ) but not in the 25 and 50 mm SCI animals (Fig. 4I). Peak detection analysis (Fig. 4J-L) demonstrated the increase in the ESA (dESA) during voiding in uninjured (Fig. $4 J$ ) and $12.5 \mathrm{~mm}$ SCI animals (Fig. $4 K$ ) but not in more severely injured animals, as illustrated by the representative animal from the $50 \mathrm{~mm}$ SCI group (Fig. $4 L$ ). The animals from the $12.5 \mathrm{~mm}$ SCI groups demonstrated dESA values that were not different from those of the uninjured group, whereas 25 and 50 mm SCI groups exhibited progressively decreased dESAs (Fig. $5 A$ ). Among the studied EUS EMG parameters, the dESA was most sensitive to SCI and was therefore used in describing detrusor-EUS coordination in subsequent analyses.

\section{Identification of spinal motoneurons innervating the bladder and the EUS}

Motoneurons innervating the EUS in the rat are located in the DL nucleus of the L6-S1 ventral horn (Schroder, 1980), and those innervating the bladder are part of the SPN (Nadelhaft and Booth, 1984). To confirm the location of DL motoneurons at the lateral portion of the ventral horn (Schroder, 1980), we injected the retrograde tracer Fluororuby in the EUS using locations similar to those in which we placed EUS EMG wires. The general histological appearance of the spinal cord sections was traced and Fluororuby labeled, and nonlabeled ventral horn motoneurons were identified (Fig. 6A, middle, $C$ ). Motoneurons that were retrogradely labeled from the EUS with Fluororuby were located in the lateral ventral horn, in the DL nucleus, over a length of $\sim 7$ $\mathrm{mm}$ from the L5-L6 junction to the L6-S1 junction. At S1, however, the morphologically identifiable DL was no longer present as a nucleus (Fig. 6B, left), and no retrogradely labeled motoneurons were present in the ventral horn (Fig. 6B, middle).

A separate animal was injected with pseudorabies virus into the bladder wall for transneuronal tracing as described previously (Pikov et al., 1998) to identify the location of bladder-projecting motoneurons in relationship to DL motoneurons. Sections through the L6 level (Fig. 6A, right) indicated a lack of pseudorabies virus labeling in DL motoneurons. However, at the S1 level there was strong labeling in the intermediolateral gray in the location of motoneurons of the SPN (Fig. 6B, right, boxed area, $D$ ).

By coexamining the neutral red-stained sections with adjacent Fluororuby sections in the DL (Fig. 6 A, circled area, left) and with the pseudorabies virus staining in the SPN (Fig. 6B, boxed area, left), the criteria for morphological identification of these areas were made. In subsequent immunohistochemical and in situ hybridization studies, the DL and SPN nuclei were identified on the basis of the neutral red-staining patterns.

\section{Relationship between detrusor-EUS coordination and preservation of long tracts after $\mathrm{SCI}$}

White matter sparing at the epicenter was significantly correlated with dESA (Fig. 5B), indicating that the degree of general supraspinal connectivity was an important factor in recovery of detrusor-EUS coordination. The preservation of descending supraspinal connections believed to be involved in the control of detrusor-EUS coordination was further probed by immunohistochemistry for long tract-descending innervation of neurons in the DL and SPN. A portion of the supraspinal-descending control innervates the DL directly (Ding et al., 1995; Hermann et al., 1998). We therefore evaluated the supraspinal terminations around the EUS-innervating motoneurons in this area using 

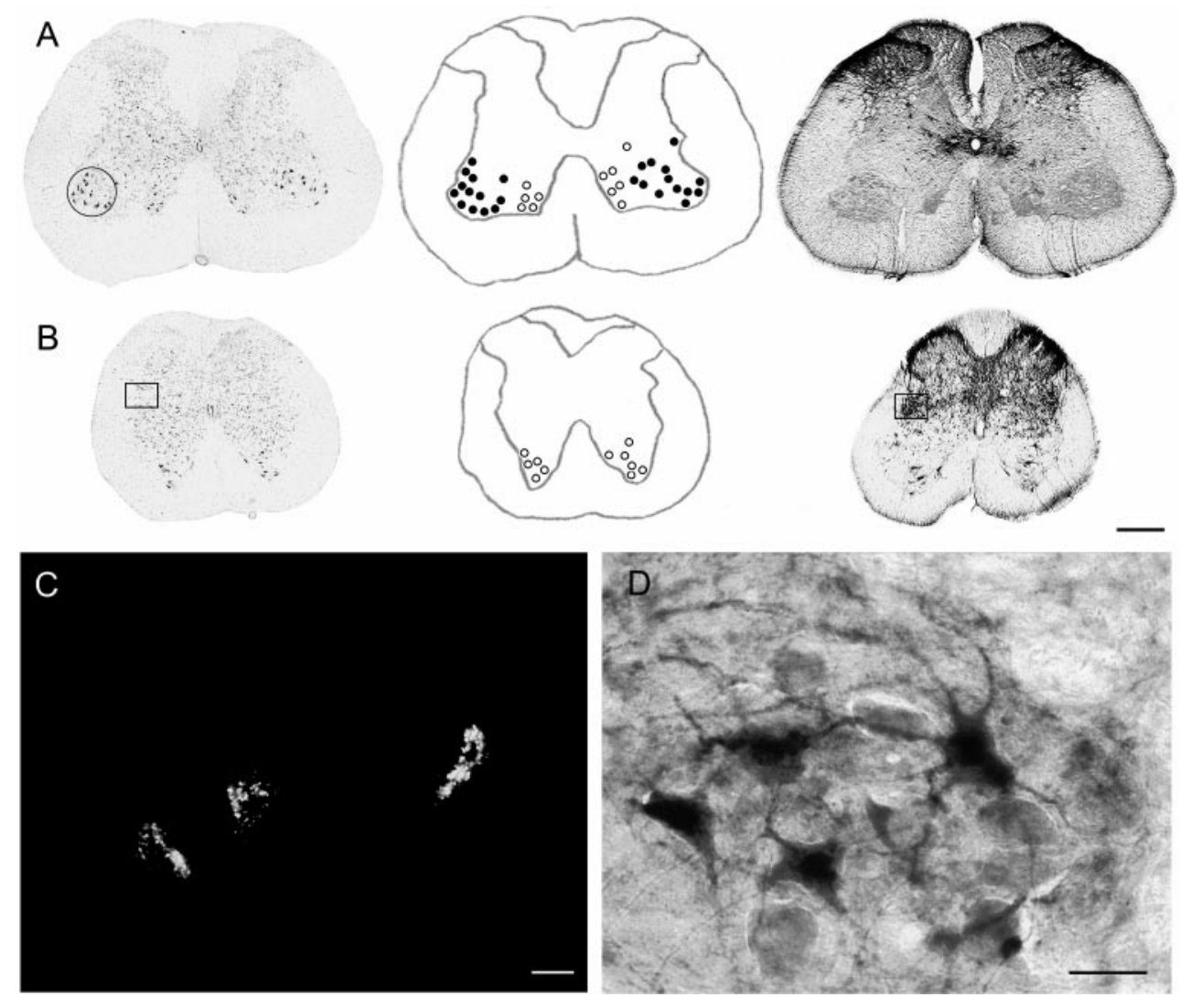

Figure 6. $A, B$, Bright-field photomicrographs and their drawings show sections through the DL nucleus at L6 $(A)$ and the SPN at S1 (B). Left, Photomicrographs show neutral red-stained neurons. Middle, Tracings of these photomicrographs show the gray matter contour and outlines of motoneurons in the ventral horn that were backlabeled with the retrograde tracer Fluororuby (closed circles) or had no labeling (open circles). Right, Photomicrographs show the pseudorabies virus tracing at the corresponding levels of spinal cord. $C$, A fluorescence photomicrograph of the area indicated by the circle in $A$ (left) shows Fluororuby deposits in DL motoneurons that were retrogradely labeled from the EUS. $D$, A bright-field photomicrograph taken from the area indicated by the box in $B$ (right) shows neurons in the SPN that were retrogradely labeled with pseudorabies virus from the bladder. Scale bars: $A, B, 500 \mu \mathrm{m}$; $C, D, 20 \mu \mathrm{m}$.

serotonin immunoreactivity, because brainstem serotonergic pathways are involved in the control of micturition (McMahon and Spillane, 1982; Durant and Yaksh, 1988; Fukuda and Koga, 1991; Thor and Katofiasc, 1995; Espey et al., 1998).

The number of neutral red-stained motoneurons in the DL was unaffected by SCI: $9.7 \pm 1.9,9.8 \pm 2.2,10.2 \pm 2.7$, and $9.8 \pm 2.0$ neurons per section for uninjured and $12.5,25$, and $50 \mathrm{~mm} \mathrm{SCI}$ groups, respectively. Similarly, the size of DL motoneurons, expressed as the cell body area, was similar in normal and SCI rats: $1113 \pm 178 \mu \mathrm{m}^{2}$ compared with $1177 \pm 153,1157 \pm 137$, and $1251 \pm 155 \mu \mathrm{m}^{2}$ in $12.5,25$, and $50 \mathrm{~mm}$ SCI groups, respectively. In uninjured rats, strong serotonin immunoreactivity was seen as a punctate stain surrounding DL motoneuronal cell bodies and their processes (Fig. 7A). All DL motoneurons demonstrated serotonergic innervation. DL motoneurons at 8 weeks after 12.5 $\mathrm{mm}$ SCI were also consistently associated with serotonergic innervation (Fig. $7 B$ ), and the density of innervation was similar to that of uninjured controls $(103 \pm 9 \%)$ based on comparison of injured and control sections on the same slide. All but 1 of $38 \mathrm{DL}$ neurons analyzed in the $25 \mathrm{~mm}$ SCI group had associated serotonin immunoreactivity, but the density (positive pixels per unit area) was significantly reduced to $25 \pm 19 \%$ of controls. In the 50 mm SCI groups many DL neurons (91 of 159) did not have associated serotonin immunoreactivity (Fig. $7 \mathrm{C}$ ), and for those that did the density was lower than that in uninjured controls $(24 \pm 18 \%)$. The average density of serotonin immunoreactivity associated with DL motoneurons in individual rats was positively correlated to their detrusor-EUS coordination as measured by dESA (Fig. 5C).

An important descending supraspinal projection to the SPN was identified using CRF immunoreactivity (Fig. $7 D-F$ ), which labels micturition- and distal colon-related pathways (Valentino et al., 1996, 2000). In uninjured rats, intense CRF immunoreactivity was associated with the SPN (Fig. 7D), and it was reduced in SCI animals (Fig. 7E,F). Quantification of CRF immunoreactivity showed an injury severity-dependent reduction chronically after SCI. As a percentage of pixel density of CRF immunoreactivity in uninjured control sections on the same slide, the SCI groups averaged $64 \pm 3,33 \pm 11$, and $21 \pm 2 \%$ for the $12.5,25$, and $50 \mathrm{~mm}$ groups, respectively. The density of CRF immunoreactivity in the SPN was positively correlated with the degree of detrusor-EUS coordination as measured by dESA (Fig. 5D).

\section{In situ hybridization analysis of glutamate receptor subunit mRNA in DL motoneurons}

In situ hybridization with oligonucleotide probes for NMDA NR1, NR2A, and NR2B and AMPA GluR1, GluR2, GluR3, and GluR4 subunit mRNAs indicated expression of these mRNAs in DL motoneurons of uninjured controls (Fig. 8). Quantification of grains showed no difference in the pattern of mRNA expressed between the uninjured animals and the animals from the $12.5 \mathrm{~mm}$ SCI group that demonstrated a normal level of detrusor-EUS coordination (Table 2). Significant increases were seen, however, in mRNA for GluR2 and NR2A in both the 25 and $50 \mathrm{~mm}$ SCI groups compared with uninjured controls or the $12.5 \mathrm{~mm}$ SCI group (Table 2). Regression analyses indicated a lack of significant correlation between levels of GluR2 or NR2A and dESA (data not shown).

\section{Relationship between recovery of LUT function and hindlimb function after graded SCI}

Injury severity had a generally similar effect on recovery of LUT function and hindlimb function. The more severe 25 and $50 \mathrm{~mm}$ SCI groups demonstrated a similar time course of recovery of 


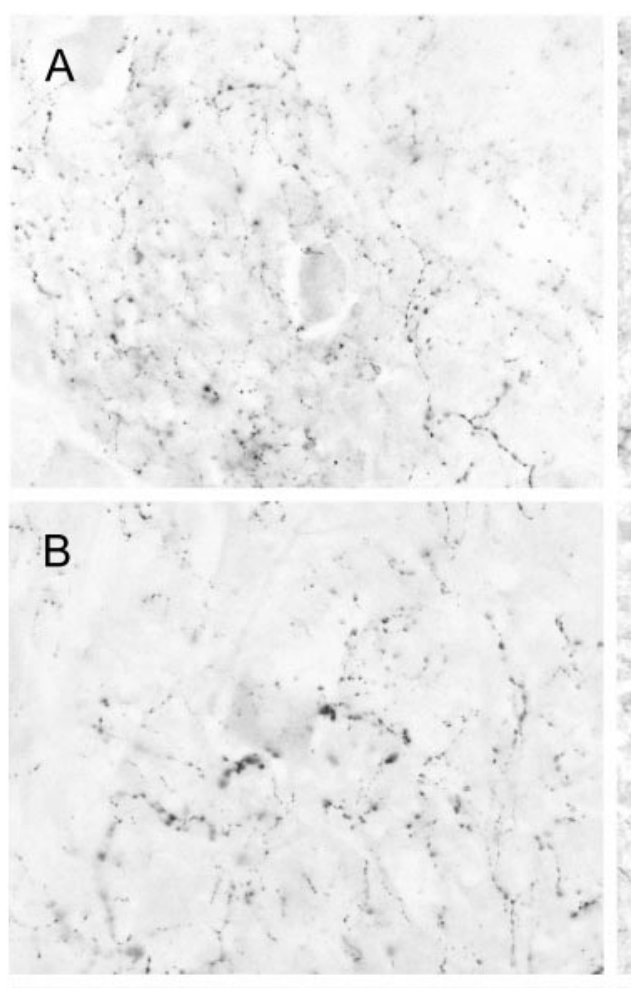

\section{C}

Figure 7. Immunoreactivity for descending projections to lumbosacral areas involved in micturition. $A-C$, Bright-field photomicrographs showing serotonin immunoreactivity in the DL. High levels of the immunoreactivity are seen in uninjured $(A)$ and $12.5 \mathrm{~mm}$ SCI $(B)$ animals, and virtually no labeling is seen in the $50 \mathrm{~mm} \mathrm{SCI}$ animal $(C) . D-F$, Bright-field photomicrographs showing CRF immunoreactivity in the SPN. Progressively decreased levels of immunoreactivity are seen in uninjured $(D), 12.5 \mathrm{~mm} \mathrm{SCI}(E)$, and $50 \mathrm{~mm}$ SCI $(F)$ animals. Scale bar, $20 \mu \mathrm{m}$.

spontaneous voiding (Fig. 3; Table 1), a similar incomplete recovery of detrusor-EUS coordination (Fig. 5A), and a similar chronic hindlimb functional deficit as measured by the CBS (Fig. $1 C$ ). Importantly, most of these animals did not recover weightbearing locomotion, as measured by the BBB (Fig. $1 B$ ), which is believed to require supraspinal control (Grillner and Dubuc, 1988; Mori et al., 1999). In contrast, the $12.5 \mathrm{~mm}$ SCI group recovered coordinated weight-bearing locomotion (Fig. $1 B$ ) and virtually normal detrusor-EUS coordination (Fig. $5 A$ ). This recovery was associated with sparing of $20 \%$ of the white matter at the injury epicenter and normal chronic levels of serotonin immunoreactivity (Fig. 6B) and glutamate receptor subunit mRNA expression associated with DL motoneurons (Table 2). The relationship between the extent of chronic LUT function and chronic hindlimb function was further examined by linear regression analysis. There was a significant correlation between CBS and dESA $\left(r^{2}=0.73 ; p<0.001\right)$ and between BBB and dESA $\left(r^{2}=\right.$ $0.71 ; p<0.001)$.

\section{DISCUSSION}

Our results demonstrate that a clinically relevant model of spinal cord contusion in the rat produces injury severity-dependent chronic effects on the coordination of urinary bladder and EUS function. Twenty percent white matter sparing at the injury epicenter was sufficient for complete recovery of detrusor-EUS coordination by 8 weeks after SCI. This robust recovery of EUS function suggests that it may be a suitable model system for studies on natural mechanisms of adaptive plasticity after SCI. In our previous study (Pikov et al., 1998), we found that partial recovery of detrusor-EUS coordination can occur by week 2 after an incomplete contusion injury in the rat. This is in contrast to results of complete transection after which detrusor-EUS coordination is permanently lost (Kruse et al., 1993; Pikov et al., 1998). Transneuronal viral tracing in our previous study (Pikov et al., 1998) indicated that the contused animals had residual connections from the bladder to brainstem centers that normally are involved in the control of micturition. We postulated that the presence of such residual connections with brainstem control centers for micturition served as the anatomical basis for the partial functional recovery. In the current study we examined this hypothesis by experimentally varying the loss of supraspinal connections by altering injury severity. We found that the degree of chronic impairment of detrusor-EUS coordination is positively 


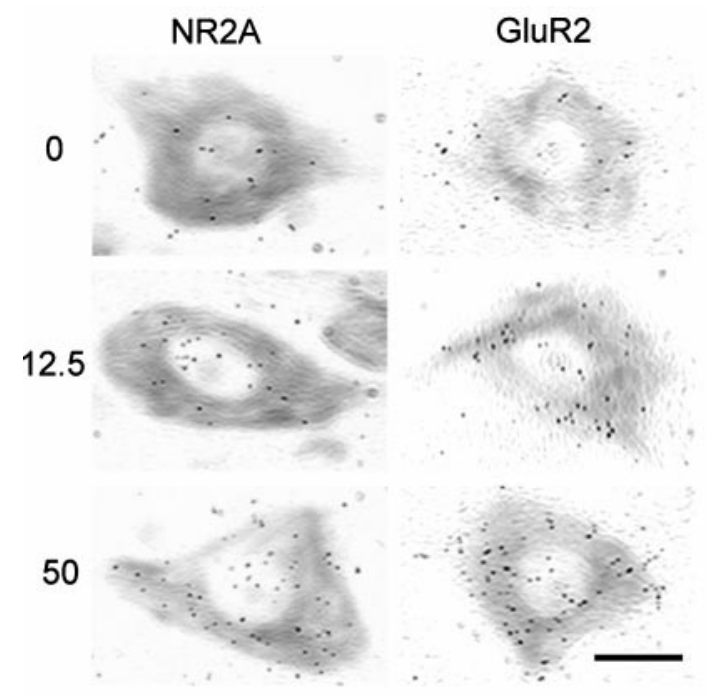

Figure 8. Bright-field photomicrographs showing neutral red-stained DL motoneurons overlaid with autoradiography grains of NMDA subunit NR2A mRNA (left) and AMPA subunit GluR2 mRNA (right). Top, An uninjured animal shows moderate levels of mRNA expression. Middle, A $12.5 \mathrm{~mm}$ SCI animal has similar levels of mRNA expression. Bottom, A 50 mm SCI animal shows high expression of both NR2A and GluR2 subunit mRNAs. Scale bar, $20 \mu \mathrm{m}$.

correlated with (1) the severity of the initial mechanical trauma, (2) the overall chronic white matter loss at the thoracic injury site, and (3) the specific loss of innervation by brainstem pathways to lower motoneurons in the lumbosacral spinal cord that innervate the bladder and the EUS. In addition, the degree of chronic detrusor-EUS coordination correlated with the recovery of the somatic hindlimb functions that also require supraspinal control, such as weight-bearing locomotion.

\section{Evaluating detrusor-EUS coordination}

For this study we applied a method of digital data analysis used previously for surface EMG data from limb muscles (Feng and Mak, 1998) to characterize quantitatively detrusor-EUS coordination in the rat. This allowed flexibility for postacquisition data filtering and analyses in time-frequency domains. The timefrequency analysis yielded important quantitative parameters, such as mean EMG activity, mean power frequency, and mean ESA. Coordinated EMG activation during the bladder contraction phase was studied in terms of timing and the amount of change in the calculated EMG parameters relative to the filling phase. The former was unaltered, and the latter was significantly affected by SCI, with ESA being the parameter that was most sensitive to injury.

\section{Role of supraspinal projections in detrusor-EUS coordination after SCI}

There are several sites in the brainstem that are implicated in detrusor-EUS coordination. The EUS-controlling motoneurons, located in the DL nucleus of L6-S1 (Schroder, 1980), receive direct and indirect (via spinal interneurons) supraspinal projections mostly from Barrington's nucleus (pontine micturition center) (Vizzard et al., 1995; Nadelhaft and Vera, 1996; Marson, 1997), the D-region just ventral to Barrington's nucleus (Ding et al., 1995), and ventrolateral pontine periaqueductal gray (Marson, 1997; Ding et al., 1998; Matsuura et al., 1998). Barrington's nucleus is likely a primary micturition-controlling center (de
Groat, 1990). Other brainstem nuclei with identified connections to bladder and EUS pathways are the raphe magnus, raphe pallidus, parapyramidal medullary reticular formation, subcoeruleus pars $\alpha$, locus coeruleus, and the A5 and A7 nuclei (Vizzard et al., 1995; Marson, 1997).

Of these, cells in the raphe nuclei and nucleus paragigantocellularis in the medullary reticular formation produce serotonin (Marson, 1997). We, therefore, used serotonin as a marker of direct supraspinal projections to DL (Ramirez-Leon et al., 1994; Tang et al., 1998). Previous studies in our laboratory and the work of others have established that serotonin immunoreactivity distal to the injury site is a good indicator of both SCI severity and the degree of recovery of somatic sensorimotor function (Faden et al., 1988; Wrathall et al., 1994; Teng and Wrathall, 1997).

CRF is expressed in cells in Barrington's nucleus that project to the SPN (Valentino et al., 1996, 2000). The SPN contains preganglionic motoneurons that innervate the bladder and proximal urethra, as well as interneurons that convey information from Barrington's nucleus to DL motoneurons (Loewy et al., 1979; Nadelhaft and Booth, 1984; Nadelhaft et al., 1986; Pascual et al., 1993; Ding et al., 1997). We used CRF immunoreactivity to assess the micturition-related supraspinal connections to the SPN.

Both of the markers of supraspinal control of micturition that we used proved to be correlated to the degree of chronic detrusor-EUS coordination. Combined, these measures indicate that recovery of detrusor-EUS coordination is associated with sparing of supraspinal projections to areas in the lumbosacral spinal cord that control bladder and EUS function. An interesting distinction is that even the most mildly injured group (12.5 mm SCI) demonstrated significant reductions in CRF innervation of the SPN, which averaged $64 \%$ of normal at 8 weeks after injury. These same rats demonstrated normal $(103 \%)$ levels of serotonin immunoreactivity associated with DL motoneurons. Approximately $80 \%$ of white matter is lost at the injury site in these animals and would be expected to include loss of serotonergic fibers from the brainstem. Indeed, unpublished data from our laboratory indicate a $40 \%$ reduction in the amount of serotonin immunoreactivity at $5 \mathrm{~d}$ after injury in $12.5 \mathrm{~mm}$ SCI rats. Therefore, sprouting and/or synaptic plasticity of spared serotonergic fibers may occur during recovery of detrusor-EUS coordination after injury to compensate for lost fibers.

\section{Role of glutamatergic transmission in normal micturition and after SCI}

Glutamate is widely distributed in both spinal and supraspinal circuits and thus cannot be readily used as a marker of supraspinal projections controlling micturition. The descending projections from Barrington's nucleus (Liu et al., 1995) described above and likely others involved in control of micturition use glutamate as a primary neurotransmitter (de Groat et al., 1998), with other substances like serotonin and CRF serving modulatory roles (Yoshiyama et al., 1994b; Pavcovich and Valentino, 1995; Hokfelt et al., 2000). It is important, therefore, to find alternative ways of studying the glutamatergic system in terms of its involvement in functional recovery after SCI. As the first effort in this direction, we investigated the pattern of ionotropic glutamate receptor subunit mRNA expression in DL motoneurons. A major finding was that the subunit expression levels in animals that recovered detrusorEUS coordination were similar to those of uninjured controls, suggesting either that SCI and subsequent recovery did not involve the alteration in NMDA or AMPA subunit receptor 
Table 2. Expression of NMDA (NR1, NR2A, and NR2B) and AMPA (GluR1, GluR2, GluR3, and GluR4) subunit mRNA in DL motoneurons at 8 weeks

\begin{tabular}{lcrrr} 
& \multicolumn{3}{l}{ SCI $(\mathrm{mm})$} & \\
\cline { 2 - 5 } Subunit & $0(n=6)$ & $12.5(n=7)$ & $25(n=3)$ & $50(n=5)$ \\
\hline NR1 & $39.4 \pm 8.7$ & $43.7 \pm 6.0$ & $43.1 \pm 6.4$ & $45.1 \pm 5.3$ \\
NR2A & $6.8 \pm 1.5$ & $9.9 \pm 2.7$ & $12.4^{*} \pm 4.1$ & $12.3^{*} \pm 2.9$ \\
NR2B & $1.6 \pm 0.4$ & $2.3 \pm 0.5$ & $2.2 \pm 0.5$ & $2.2 \pm 0.2$ \\
GluR1 & $1.3 \pm 0.2$ & $1.6 \pm 0.5$ & $1.6 \pm 0.4$ & $2.0 \pm 0.6$ \\
GluR2 & $4.6 \pm 1.1$ & $6.3 \pm 2.2$ & $10.5^{*} \pm 1.6$ & $8.2^{*} \pm 2.6$ \\
GluR3 & $3.2 \pm 2.1$ & $5.8 \pm 1.3$ & $4.9 \pm 1.6$ & $5.9 \pm 2.6$ \\
GluR4 & $2.4 \pm 0.6$ & $2.9 \pm 0.6$ & $2.0 \pm 0.3$ & $2.3 \pm 0.2$ \\
\hline
\end{tabular}

The data are presented as the number of grains per square micrometer of cell area. Significant difference from the uninjured group is indicated by an asterisk and is based on $p<0.05$ in Tukey's post hoc test after ANOVA.

patterns in DL motoneurons or that during recovery the receptor subunit patterns returned to normal values after an initial perturbation. In contrast, animals without recovery of detrusor-EUS coordination showed elevated levels of specific NMDA and AMPA subunits. If these changes in chronic mRNA levels are assumed to produce altered functional glutamate receptors, these alterations may be related to the aberrant hyperactivity of these motoneurons as seen in the spontaneous spastic activity chronically in lumbosacral motoneurons after SCI (Hiersemenzel et al., 2000; Little et al., 2000).

It should be noted that the absence of altered mRNA patterns in DL motoneurons in the rats that recovered detrusor-EUS coordination does not preclude a role of glutamatergic systems in this functional recovery. For example, significant alterations may take place in glutamatergic sensory, brainstem, and/or spinal cord interneuronal portions of the pathways involved or may involve other potential alterations in DL motoneurons that we did not study, such as altered expression of subunit splice variants that can occur after SCI (Prybylowski et al., 2001).

\section{Adaptive plasticity in control of micturition: lessons from development}

The establishment of supraspinal control of micturition during postnatal development involves adaptive synaptic plasticity. Studies by de Groat and colleagues (Kruse et al., 1993; de Groat et al., 1998) indicate that shortly after birth, animals undergo a switch from spinal to supraspinal control mediating the detrusor-EUS voiding reflex that persists through adulthood. This switch involves an activity-dependent competition between spinal and supraspinal inputs at individual synapses. It is possible therefore that recovery of detrusor-EUS coordination after incomplete SCI involves a similar adaptive activity-dependent mechanism. Continued recovery of voluntary EUS function weeks and months after SCI in humans (Schurch, 1999) suggests that there is an ongoing process of greater use of spared control. Because normal EUS control is mediated mostly by glutamatergic neurotransmission (de Groat et al., 1998), an improved coordination chronically after SCI may involve an adaptation in glutamatergic neurotransmission in the function of preserved supraspinal control. This hypothesis will be tested in our future experiments.

In conclusion, our results indicate that the recovery of detrusor-EUS coordination after SCI occurs in an injury severitydependent manner. This functional recovery may offer a suitable model system to study the adaptive plasticity of residual supraspinal connections after SCI. Thorough pharmacological investiga- tion of the key neurotransmitters and their receptors involved in the normal and recovered detrusor-EUS coordination may provide clues regarding natural mechanisms of recovery of supraspinal control. This information may serve as the basis of pharmacological and/or other strategies to enhance functional recovery after SCI.

\section{REFERENCES}

Basso DM, Beattie MS, Bresnahan JC (1995) A sensitive and reliable locomotor rating scale for open field testing in rats. J Neurotrauma $12: 1-21$.

Basso DM, Beattie MS, Bresnahan JC (1996) Graded histological and locomotor outcomes after spinal cord contusion using the NYU weightdrop device versus transection. Exp Neurol 139:244-256.

Beattie MS, Bresnahan JC, Komon J, Tovar CA, Van Meter M, Anderson DK, Faden AI, Hsu CY, Noble LJ, Salzman S, Young W (1997) Endogenous repair after spinal cord contusion injuries in the rat. Exp Neurol 148:453-463.

Bracken MB, Shepard MJ, Collins WF, Holford TR, Young W, Baskin DS, Eisenberg HM, Flamm E, Leo-Summers L, Maroon J, Marshall LF, Perot PL, Piepmeier J, Sonntag VKH, Wagner FC, Wilberger JE, Winn HR (1990) A randomized, controlled trial of methylprednisolone or naloxone in the treatment of acute spinal-cord injury. Results of the Second National Acute Spinal Cord Injury Study. N Engl J Med 322:1405-1411.

Callsen-Cencic P, Mense S (1998) Abolition of cystitis-induced bladder instability by local spinal cord cooling. J Urol 160:236-241.

Clark G (1981) Chromoxane cyanine R. In: Staining procedures, 4th Edition (Clark G, ed), pp 109-110. Baltimore: Williams and Wilkins.

Constantini S, Young W (1994) The effects of methylprednisolone and the ganglioside GM1 on acute spinal cord injury in rats. J Neurosurg 80:97-111.

de Groat WC (1990) Central neural control of the lower urinary tract. Ciba Found Symp 151:27-44.

de Groat WC (1995) Mechanisms underlying the recovery of lower urinary tract function following spinal cord injury. Paraplegia 33:493-505.

de Groat WC, Araki I, Vizzard MA, Yoshiyama M, Yoshimura N, Sugaya K, Tai C, Roppolo JR (1998) Developmental and injury induced plasticity in the micturition reflex pathway. Behav Brain Res 92:127-140.

Dietz V, Nakazawa K, Wirz M, Erni T (1999) Level of spinal cord lesion determines locomotor activity in spinal man. Exp Brain Res 128:405-409.

Ding YQ, Takada M, Tokuno H, Mizuno N (1995) Direct projections from the dorsolateral pontine tegmentum to pudendal motoneurons innervating the external urethral sphincter muscle in the rat. J Comp Neurol 357:318-330.

Ding YQ, Zheng HX, Gong LW, Lu Y, Zhao H, Qin BZ (1997) Direct projections from the lumbosacral spinal cord to Barrington's nucleus in the rat: a special reference to micturition reflex. J Comp Neurol 389:149-160.

Ding YQ, Wang D, Nie H, Guan ZL, Lu BZ, Li JS (1998) Direct projections from the periaqueductal gray to pontine micturition center neurons projecting to the lumbosacral cord segments: an electron microscopic study in the rat. Neurosci Lett 242:97-100.

Durant PA, Yaksh TL (1988) Micturition in the unanesthetized rat: effects of intrathecal capsaicin, $N$-vanillylnonanamide, 6-hydroxydopamine and 5,6-dihydroxytryptamine. Brain Res 451:301-308. 
Espey MJ, Du HJ, Downie JW (1998) Serotonergic modulation of spinal ascending activity and sacral reflex activity evoked by pelvic nerve stimulation in cats. Brain Res 798:101-108.

Faden AI, Gannon A, Basbaum AI (1988) Use of serotonin immunocytochemistry as a marker of injury severity after experimental spinal trauma in rats. Brain Res 450:94-100.

Fedirchuk B, Shefchyk SJ (1991) Effects of electrical stimulation of the thoracic spinal cord on bladder and external urethral sphincter activity in the decerebrate cat. Exp Brain Res 84:635-642.

Feng CJ, Mak AF (1998) EMG analysis of voluntary elbow movements in subjects with spasticity. Electromyogr Clin Neurophysiol 38:393-404.

Fukuda H, Koga T (1991) Midbrain stimulation inhibits the micturition, defecation and rhythmic straining reflexes elicited by activation of sacral vesical and rectal afferents in the dog. Exp Brain Res 83:303-316.

Gale K, Kerasidis H, Wrathall JR (1985) Spinal cord contusion in the rat: behavioral analysis of functional neurologic impairment. Exp Neurol 88:123-134.

Grillner S, Dubuc R (1988) Control of locomotion in vertebrates: spinal and supraspinal mechanisms. Adv Neurol 47:425-453.

Grossman SD, Wolfe BB, Yasuda RP, Wrathall JR (1999) Alterations in AMPA receptor subunit expression after experimental spinal cord contusion injury. J Neurosci 19:5711-5720.

Grossman SD, Wolfe BB, Yasuda RP, Wrathall JR (2000) Changes in NMDA receptor subunit expression in response to contusive spinal cord injury. J Neurochem 75:174-184.

Gruner JA (1992) A monitored contusion model of spinal cord injury in the rat. J Neurotrauma 9:123-126; discussion 126-128.

Hermann GE, Bresnahan JC, Holmes GM, Rogers RC, Beattie MS (1998) Descending projections from the nucleus raphe obscurus to pudendal motoneurons in the male rat. J Comp Neurol 397:458-474.

Hiersemenzel LP, Curt A, Dietz V (2000) From spinal shock to spasticity: neuronal adaptations to a spinal cord injury. Neurology 54:1574-1582

Hokfelt T, Arvidsson U, Cullheim S, Millhorn D, Nicholas AP, Pieribone V, Seroogy K, Ulfhake B (2000) Multiple messengers in descending serotonin neurons: localization and functional implications. J Chem Neuroanat 18:75-86.

Kakizaki H, Fraser MO, de Groat WC (1997) Reflex pathways controlling urethral striated and smooth muscle function in the male rat. Am J Physiol 272:R1647-R1656.

Kerasidis H, Wrathall JR, Gale K (1987) Behavioral assessment of functional deficit in rats with contusive spinal cord injury. J Neurosci Methods 20:167-179.

Ko HY, Ditunno Jr JF, Graziani V, Little JW (1999) The pattern of reflex recovery during spinal shock. Spinal Cord 37:402-409.

Kruse MN, Noto H, Roppolo JR, de Groat WC (1990) Pontine control of the urinary bladder and external urethral sphincter in the rat. Brain Res 532:182-190.

Kruse MN, Belton AL, de Groat WC (1993) Changes in bladder and external urethral sphincter function after spinal cord injury in the rat. Am J Physiol 264:R1157-R1163.

Leis AA, Kronenberg MF, Stetkarova I, Paske WC, Stokic DS (1996) Spinal motoneuron excitability after acute spinal cord injury in humans. Neurology 47:231-237.

Little JW, Burns SP, James JJ, Stiens SA (2000) Neurologic recovery and neurologic decline after spinal cord injury. Phys Med Rehabil Clin N Am 11:73-89.

Liu RH, Fung SJ, Reddy VK, Barnes CD (1995) Localization of glutamatergic neurons in the dorsolateral pontine tegmentum projecting to the spinal cord of the cat with a proposed role of glutamate on lumbar motoneuron activity. Neuroscience 64:193-208.

Loewy AD, Saper CB, Baker RP (1979) Descending projections from the pontine micturition center. Brain Res 172:533-538.

Maggi CA, Giuliani S, Santicioli P, Meli A (1986a) Analysis of factors involved in determining urinary bladder voiding cycle in urethananesthetized rats. Am J Physiol 251:R250-R257.

Maggi CA, Santicioli P, Meli A (1986b) The nonstop transvesical cystometrogram in urethane-anesthetized rats: a simple procedure for quantitative studies on the various phases of urinary bladder voiding cycle. J Pharmacol Methods 15:157-167.

Marson L (1997) Identification of central nervous system neurons that innervate the bladder body, bladder base, or external urethral sphincter of female rats: a transneuronal tracing study using pseudorabies virus. J Comp Neurol 389:584-602.

Matsuura S, Allen GV, Downie JW (1998) Volume-evoked micturition reflex is mediated by the ventrolateral periaqueductal gray in anesthetized rats. Am J Physiol 275:R2049-R2055.

McMahon SB, Spillane K (1982) Brain stem influences on the parasympathetic supply to the urinary bladder of the cat. Brain Res 234:237-249.

Mersdorf A, Schmidt RA, Tanagho EA (1993) Urodynamic evaluation and electrical and pharmacologic neurostimulation. The rat model. Urol Res 21:199-209.

Mori S, Matsui T, Kuze B, Asanome M, Nakajima K, Matsuyama K
(1999) Stimulation of a restricted region in the midline cerebellar white matter evokes coordinated quadrupedal locomotion in the decerebrate cat. J Neurophysiol 82:290-300.

Morrison JF (1997) Central nervous control of the bladder. In: Central nervous control of autonomic function (Jordan D, ed), pp 129-149. Amsterdam: Harwood Academic.

Nadelhaft I, Booth AM (1984) The location and morphology of preganglionic neurons and the distribution of visceral afferents from the rat pelvic nerve: a horseradish peroxidase study. J Comp Neurol 226:238-245.

Nadelhaft I, Vera PL (1996) Neurons in the rat brain and spinal cord labeled after pseudorabies virus injected into the external urethral sphincter. J Comp Neurol 375:502-517.

Nadelhaft I, de Groat WC, Morgan C (1986) The distribution and morphology of parasympathetic preganglionic neurons in the cat sacral spinal cord as revealed by horseradish peroxidase applied to the sacral ventral roots. J Comp Neurol 249:48-56.

Noble LJ, Wrathall JR (1985) Spinal cord contusion in the rat: morphometric analyses of alterations in the spinal cord. Exp Neurol 88:135-149.

Pascual JI, Insausti R, Gonzalo LM (1993) Urinary bladder innervation in male rat: termination of primary afferents in the spinal cord as determined by transganglionic transport of WGA-HRP. J Urol 150:500-504

Pavcovich LA, Valentino RJ (1995) Central regulation of micturition in the rat the corticotropin-releasing hormone from Barrington's nucleus. Neurosci Lett 196:185-188.

Pikov V, Gillis RA, Jasmin L, Wrathall JR (1998) Assessment of lower urinary tract functional deficit in rats with contusive spinal cord injury. J Neurotrauma 15:375-386.

Prybylowski KL, Grossman SD, Wrathall JR, Wolfe BB (2001) Expression of splice variants of the NR1 subunit of the NMDA receptor in the normal and injured rat spinal cord. $J$ Neurochem, in press.

Ramirez-Leon V, Ulfhake B, Arvidsson U, Verhofstad AA, Visser TJ, Hokfelt T (1994) Serotoninergic, peptidergic and GABAergic innervation of the ventrolateral and dorsolateral motor nuclei in the cat S1/S2 segments: an immunofluorescence study. J Chem Neuroanat 7:87-103.

Schroder HD (1980) Organization of the motoneurons innervating the pelvic muscles of the male rat. J Comp Neurol 192:567-587.

Schurch B (1999) The predictive value of plantar flexion of the toes in the assessment of neuropathic voiding disorders in patients with spine lesions at the thoracolumbar level. Arch Phys Med Rehabil 80:681-686.

Selzman AA, Hampel N (1993) Urologic complications of spinal cord injury. Urol Clin North Am 20:453-464.

Tang Y, Rampin O, Calas A, Facchinetti P, Giuliano F (1998) Oxytocinergic and serotonergic innervation of identified lumbosacral nuclei controlling penile erection in the male rat. Neuroscience 82:241-254.

Teng YD, Wrathall JR (1997) Local blockade of sodium channels by tetrodotoxin ameliorates tissue loss and long-term functional deficits resulting from experimental spinal cord injury. $\mathrm{J}$ Neurosci 17:4359-4366.

Thor KB, Katofiasc MA (1995) Effects of duloxetine, a combined serotonin and norepinephrine reuptake inhibitor, on central neural control of lower urinary tract function in the chloralose-anesthetized female cat. J Pharmacol Exp Ther 274:1014-1024.

Tiseo PJ, Yaksh TL (1990) The spinal pharmacology of urinary function: studies on urinary continence in the unanaesthetized rat. Ciba Found Symp 151:91-104.

Valentino RJ, Chen S, Zhu Y, Aston-Jones G (1996) Evidence for divergent projections to the brain noradrenergic system and the spinal parasympathetic system from Barrington's nucleus. Brain Res 732:1-15.

Valentino RJ, Kosboth M, Colflesh M, Miselis RR (2000) Transneuronal labeling from the rat distal colon: anatomic evidence for regulation of distal colon function by a pontine corticotropin-releasing factor system. J Comp Neurol 417:399-414.

Vizzard MA, Erickson VL, Card JP, Roppolo JR, de Groat WC (1995) Transneuronal labeling of neurons in the adult rat brainstem and spinal cord after injection of pseudorabies virus into the urethra. J Comp Neurol 355:629-640.

Wrathall JR, Choiniere D, Teng YD (1994) Dose-dependent reduction of tissue loss and functional impairment after spinal cord trauma with the AMPA/kainate antagonist NBQX. J Neurosci 14:6598-6607.

Wrathall JR, Li W, Hudson LD (1998) Myelin gene expression after experimental contusive spinal cord injury. J Neurosci 18:8780-8793.

Yoshiyama M, Roppolo JR, De Groat WC (1994a) Alteration by urethane of glutamatergic control of micturition. Eur $\mathrm{J}$ Pharmacol 264:417-425

Yoshiyama M, Roppolo JR, de Groat WC (1994b) Interactions between glutamatergic and monoaminergic systems controlling the micturition reflex in the urethane-anesthetized rat. Brain Res 639:300-308.

Yoshiyama M, Nezu FM, Yokoyama O, de Groat WC, Chancellor MB (1999) Changes in micturition after spinal cord injury in conscious rats. Urology 54:929-933. 